\title{
Time-resolved ARPES at LACUS: Band Structure and Ultrafast Electron Dynamics of Solids
}

\author{
Alberto Crepaldia, Silvan Rotha, Gianmarco Gattia, Christopher A. Arrell ${ }^{\mathrm{b}}$, José Ojeda ${ }^{\mathrm{b}}$, Frank van \\ Mourik $^{\mathrm{b}}$, Philippe Bugnon ${ }^{\mathrm{a}}$, Arnaud Magrez ${ }^{\mathrm{a}}$, Helmuth Berger ${ }^{\mathrm{a}}$, Majed Cherguib, and Marco Grioni ${ }^{\star a}$
}

\begin{abstract}
The manipulation of the electronic properties of solids by light is an exciting goal, which requires knowledge of the electronic structure with energy, momentum and temporal resolution. Time- and angle-resolved photoemission spectroscopy (tr-ARPES) is the most direct probe of the effects of an optical excitation on the band structure of a material. In particular, tr-ARPES in the extreme ultraviolet (VUV) range gives access to the ultrafast dynamics over the entire Brillouin zone. VUV tr-ARPES experiments can now be performed at the ASTRA (ARPES Spectrometer for Time-Resolved Applications) end station of Harmonium, at LACUS. Its capabilities are illustrated by measurements of the ultrafast electronic response of ZrSiTe, a novel topological semimetal characterized by linearly dispersing states located at the Brillouin zone boundary.
\end{abstract}

Keywords: Angle-resolved photo emission spectroscopy (ARPES) · LACUS · ZrSiTe

Angle-resolved photoemission spectroscopy (ARPES) is a powerful probe of the electronic structure of solids. ${ }^{[1]}$ It yields the energy-momentum band dispersion, and provides fundamental microscopic information on the electronic correlations and electron-lattice interactions that shape the properties of interesting materials. ${ }^{[2]}$ Moreover, in pump-probe experiments it gives access to the electron dynamics in the time domain. ${ }^{[3]}$ In ARPES, the dispersion of the electronic states $E(k)$ is encoded in the angular dependence of the kinetic energy $E_{\text {kin }}$ of the photoelectrons, through the equations that express the conservation of energy and momentum:

$$
\begin{aligned}
E_{B} & =h \nu-E_{k i n}-\phi_{W F} ; \\
k_{\|} & =\frac{1}{\hbar} \sqrt{2 m_{e} E_{k i n}} \sin \theta .
\end{aligned}
$$

\footnotetext{
${ }^{\star}$ Correspondence: Prof. Dr. M. Grionia

E-mail: marco.grioni@epfl.ch

anstitute of Physics

Ecole Polytechnique Fédérale de Lausanne (EPFL) $\mathrm{CH}-1015$ Lausanne

bLaboratory of Ultrafast Spectroscopy, ISIC

and Lausanne Centre for Ultrafast Science (LACUS)

Ecole Polytechnique Fédérale de Lausanne (EPFL)

$\mathrm{CH}-1015$ Lausanne
}

$E_{B}$ and $m_{e}$ are the electron binding energy and mass, $h v$ is the photon energy, $\phi_{W F}$ is the material's work function, and $\theta$ the emission angle with respect to the sample's surface normal. $k \|=\left(k_{x} ; k_{y}\right)$ is the surface projection of the electron's momentum which, unlike the third component $k_{z}$, is conserved in the photoemission process.

Parallel momentum conservation, and a large surface sensitivity, makes ARPES an ideal technique to study the band structure of surfaces and, more generally, of twodimensional (2D) materials..$^{[4,5]}$ ARPES experiments were for instance instrumental to verify theoretical predictions of linearly dis- persing electronic states (Dirac cones) at the Fermi level $\left(\mathrm{E}_{\mathrm{F}}\right)$ of graphene. These peculiar states are solutions of the Dirac equation ${ }^{[6]}$ with the speed of light being replaced by the Fermi velocity $\mathrm{v}_{\mathrm{F}} \approx 0: 003$ c. ${ }^{[7,8]}$ The linear crossing of the bands, without the formation of energy gaps, reflects the symmetry of the graphene honeycomb lattice (Fig. 1(a)), where the rhombic unit cell contains two nonequivalent carbon atoms $\mathrm{C}_{\mathrm{A}}$ and $\mathrm{C}_{\mathrm{B}}{ }^{\cdot{ }^{[4]}}$ The according reciprocal space picture is shown in Fig. 1(b). ARPES measurements reveal the linearly dispersing Dirac cones at the $\mathrm{K}$ and $\mathrm{K}$ ' corners of the hexagonal Brillouin zone (BZ), as shown in the inset.

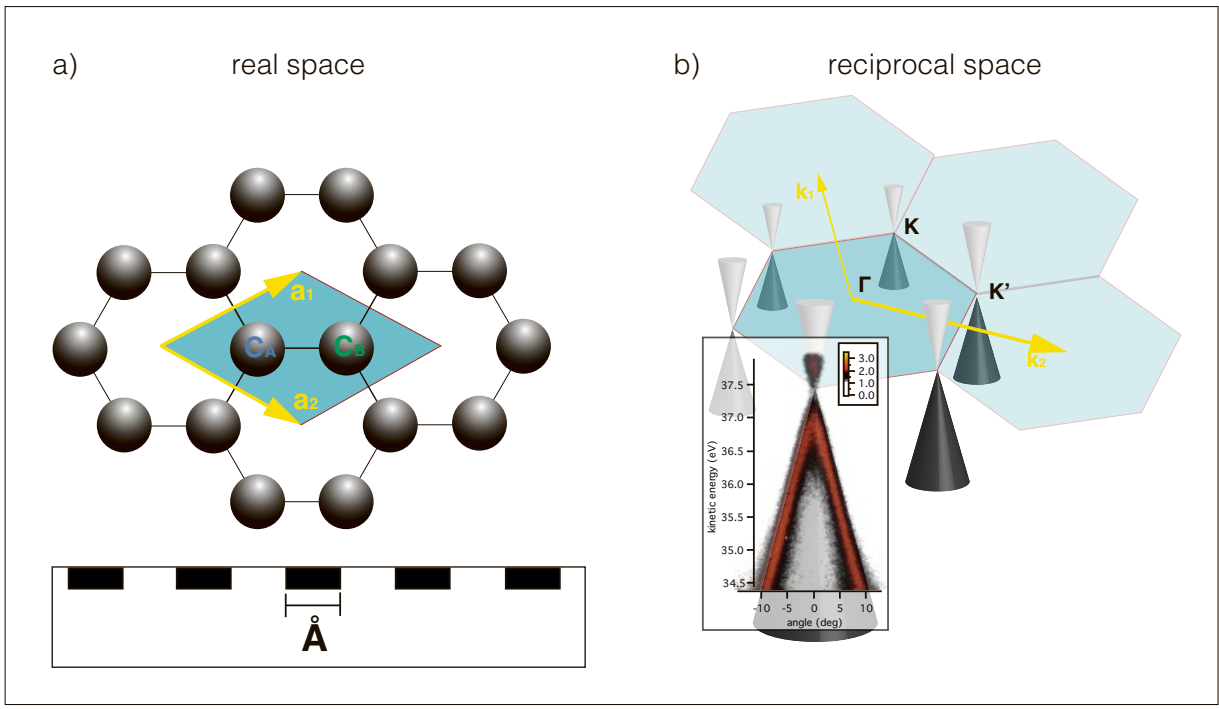

Fig. 1. (a) Real space sketch of graphene. The unit cell (central rhomb) contains two carbon atoms $\mathrm{C}_{\mathrm{A}}$, and $\mathrm{C}_{\mathrm{B}}$, one from each sublattice. (b) Corresponding reciprocal space picture with the first Brillouin zone (BZ) in the center. The electronic band structure around $E_{F}$ consists of six double cones touching at the $\mathrm{K}$ and $\mathrm{K}^{\prime}$ points of the BZ. ARPES measurement of a $\mathrm{p}$-doped graphene sample showing a branch of one of the Dirac cones. 
The concept of Dirac fermions, as low energy quasiparticle excitations in solids, extends far beyond graphene. Spinpolarized Dirac particles have been observed at the surface of topological insulators (TIs). ${ }^{[9]}$ Three-dimensional (3D) Dirac particles have been discovered in bulk crystals, where the linear dispersion in all three momentum directions is topologically protected by the presence of rotational symmetries. ${ }^{[10,11]}$ The interest in these novel states of quantum matter is not purely academic. It also stems from the prospect of achieving defect-tolerant spin currents in the former, ${ }^{[12]}$ and ultrahigh mobilities in the latter. ${ }^{[11]}$ Besides the transport properties, the linear dispersion of the 2D and 3D Dirac particles accounts also for some unconventional optical properties. ${ }^{[13,14]}$ Light-harvesting[15] and optically induced spin-[16] and valley-polarized ${ }^{[17,18]}$ excitations are amongst the perspectives opened by the capability of manipulating the band structure by an optical excitation.

ARPES has recently experienced a fast development towards experiments enabling simultaneous energy, momentum and temporal resolution. The goal is to track transient changes of the electronic population induced by an optical excitation. Time-resolved ARPES (tr-ARPES) requires pulsed laser sources providing ultrashort pulses with duration $\approx 100 \mathrm{fs}$ ( $\left.1 \mathrm{fs}=10^{-15} \mathrm{~s}\right)$. The temporal evolution of the band structure is mapped in a stroboscopic pump-probe experimental scheme, by varying the difference in optical path between the two pulses. The optical excitation (pump) often exploits the fundamental wavelength of the laser, whereas the photon energy of the probe pulse must be larger than the work function $\phi_{\mathrm{wF}}$. The possibility of optically generating spin currents in the surface Dirac particle of TIs has also motivated tr-ARPES studies, ${ }^{[19-33]}$ also with spin resolution ${ }^{[34-37]}$ aimed at understanding the out-of-equilibrium scattering mechanisms.

The momentum window of an ARPES measurement is determined by the photon energy. According to Eqn. (2) the largest accessible electron wave vector $\mathrm{k}_{\max }$ (for $E_{B}$ $\left.=0 ; \theta=90^{\circ}\right)$ is proportional to $\sqrt{h v-\phi_{W F}}$. For low photon energies $(5.9-6.3 \mathrm{eV})$ corresponding to the $4^{\text {th }}$ harmonic generated in non-linear crystals, and for typical values $\phi_{\mathrm{WF}} 4-5 \mathrm{eV}$, this yields $\mathrm{k}_{\max }<0.6 \AA^{-1}$. Yet smaller values are obtained for more realistic emission angles $\left(\theta_{\max } \approx 60^{\circ}\right)$. In TIs, the Dirac cone is located at the center of the surface-projected Brillouin zone (SBZ), and the relatively small Fermi wavevector $\left(\mathrm{k}_{\mathrm{F}} \approx 0.2^{\circ} \AA^{-1}\right)$ is well within this window. By contrast, the Dirac cones of graphene (Fig. 1) are centered at $\mathrm{k}_{\mathrm{F}}=1.7 \AA^{-1}$, which requires photon energies larger than $h v \geq$ $16 \mathrm{eV}$. Therefore the hot carrier dynam- ics of quasi-free standing single-layer ${ }^{[38,39]}$ and bilayer ${ }^{[40]}$ graphene could only be explored using high harmonics generation (HHG) in a gas. Those studies have clarified the complex relaxation mechanisms including scattering with electrons, with hot phonons ${ }^{[41]}$ and with lattice defects. ${ }^{[38]}$ They also showed that multiple hot electrons can be generated in the unoccupied part of the Dirac cone as a result of charge carrier multiplication, an effect of great interest in the perspective of an efficient conversion of light into electrical current. ${ }^{[42]}$

The capability of tr-ARPES in the extreme ultraviolet (VUV) photon energy range, to map the out-of-equilibrium electron dynamics over the entire $\mathrm{BZ}$ is of pivotal importance for many interesting systems, such as the transition metal dichalcogenides ${ }^{[43,44]}$ and perovskites. ${ }^{[45-47]}$ It is also crucial to resolve the antinodal excitations in the cuprate high-temperature superconductors. ${ }^{[48]}$ However, owing to the technical difficulty of achieving high flux and stable harmonic generation, only a few VUV tr-ARPES instruments are operational worldwide. In this article we present the results of the first ARPES and tr-ARPES experiments carried out at ASTRA (ARPES Spectrometer for Time-Resolved Applications) end station of Harmonium, ${ }^{[49]}$ at the Lausanne centre for ultrafast science (LACUS), EPFL. We show that the large momentum range made accessible by the HHG source allows us to determine the dispersion of the spin-orbit split Dirac particles at the zone boundary of the novel nodal line Dirac semimetal ZrSiTe. ${ }^{[50-52]}$ We also show preliminary data on the ultrafast out-of-equilibrium dynamics in this material.

A detailed description of the laser source and of the HHG setup can be found in ref. [49] and in the accompanying article 'Harmonium: An Ultrafast Vacuum Ultraviolet Facility' ${ }^{[53]}$ in this issue. In the following we provide more technical information about the ASTRA end station. In the present study we have employed the $17^{\text {th }}$ to $21^{\text {st }}$ harmonics generated in argon, covering the $26-33 \mathrm{eV}$ photon energy range. The linear polarization of the HHG can be switched between vertical $(\mathrm{V})$ and horizontal $(\mathrm{H})$, which allows us to take advantage of polarization-dependent matrix elements in the photoemission process, as shown later. The HHG probe beam is focused on the surface of the sample, placed in an ultrahigh vacuum (UHV) environment, to a spot size smaller than $100 \mathrm{x}$ $100 \mu \mathrm{m}^{2}$. Photoelectrons are collected and analyzed by a Specs Phoibos 150 electrostatic hemispherical analyzer, with an angular resolution $\Delta \theta=0.3^{\circ}$ and an energy resolution that can be varied between $\Delta \mathrm{E}$ $=10 \mathrm{meV}$ for static ARPES and $120 \mathrm{meV}$ for tr-ARPES experiments. The bandwidth of the HHG pulses was estimated from the total experimental broadening of the Fermi-Dirac distribution in low temperature experiments to be $<100 \mathrm{meV}$. This energy broadening corresponds well to the temporal duration of $240 \mathrm{fs}$, measured for the grating used in this work (900 gr/ $\mathrm{mm}) .{ }^{[49]}$ The UHV photoemission chamber is also equipped with a monochromatized Gammadata VUV5000 He electron cyclotron resonance (ECR) source, providing high-brilliance $\mathrm{He} \mathrm{I} \alpha(21.2 \mathrm{eV})$ and $\mathrm{He}$ II $\alpha(40.8 \mathrm{eV})$ emission lines. This source is used in high-energy resolution static ARPES measurements to determine the band structure prior to the tr-ARPES experiments. The pump at $800 \mathrm{~nm}$ was $\mathrm{H}$ polarized and the fluence on the sample surface was $\approx 0.5 \mathrm{mJcm}^{-1}$. High quality single crystals of $\mathrm{ZrSiTe}$ were produced by the chemical vapor transport method and cleaved in situ to expose atomically clean surfaces.

In the following we present preliminary results on the novel topological semimetal $\mathrm{ZrSiTe}$, which illustrate the capabilities of ASTRA for studies of the equilibrium and the ultrafast electron dynamics of solids. The crystal structure of $\mathrm{ZrSiTe}$ (Fig. 2(a)) belongs to the tetragonal $P 4 / \mathrm{nmm}$ space group, ${ }^{[52]}$ with lattice parameters $\mathrm{a}=3.7$ $\AA$ and $\mathrm{c}=9.5 \AA{ }^{[54]}$ It results from the ordered stacking of Te (brown), Zr (green) and $\mathrm{Si}$ (blue) planes in quintuple layers. The weak van der Waals interaction between two adjacent Te layers determines the most favorable cleavage plane. Fig. 2(b) shows the corresponding 3D BZ with the high-symmetry points, and the SBZ for the (001) cleavage surface.

$\mathrm{ZrSiTe}$ and its sister compounds $\mathrm{ZrSiS}$ and $\mathrm{ZrSiSe}$ have recently attracted considerable attention as members of the novel topological family of nodal line Dirac semimetals (NSMs). ${ }^{[50-52,55,56]}$ The hallmark of these materials is schematically illustrated in Fig. 2(c). In 2D and 3D Dirac semimetals, such as graphene and $\mathrm{Cd}_{3} \mathrm{As}_{2}$, the linear dispersion gives rise to pointlike Fermi surfaces, in correspondence of the Dirac points, DP (top). By contrast, in NSMs the linearly dispersing states cross not only at isolated points, but along a continuous line in reciprocal space. Hence, for a suitable doping level, the Fermi surface is formed by a closed line resulting from the crossing of the inverted valence (green) and conduction (yellow) bands (bottom). The presence of a nodal line of Dirac points has important consequences on the transport properties, such as the emergence of a large non-saturating magnetoresistance. ${ }^{[51,55,56]}$

Fig. 3 presents an overview of the band structure of $\mathrm{ZrSiTe}$ obtained by means of static ARPES. Fig. 3(a) shows the Fermi surface measured with the He I emission 
(a)

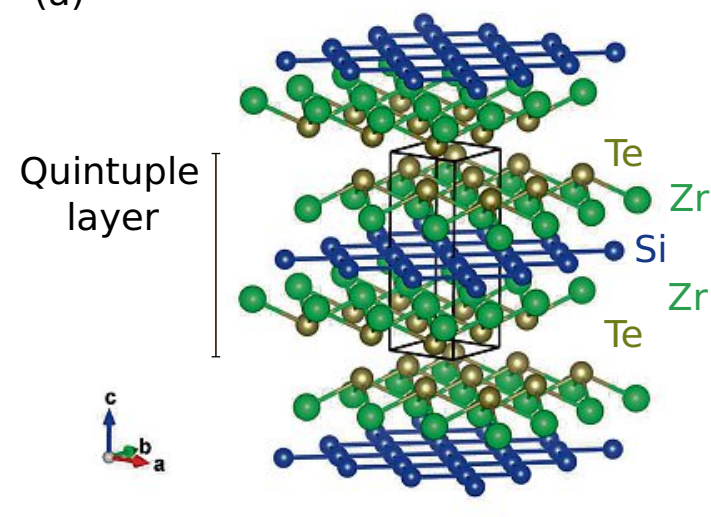

(b)

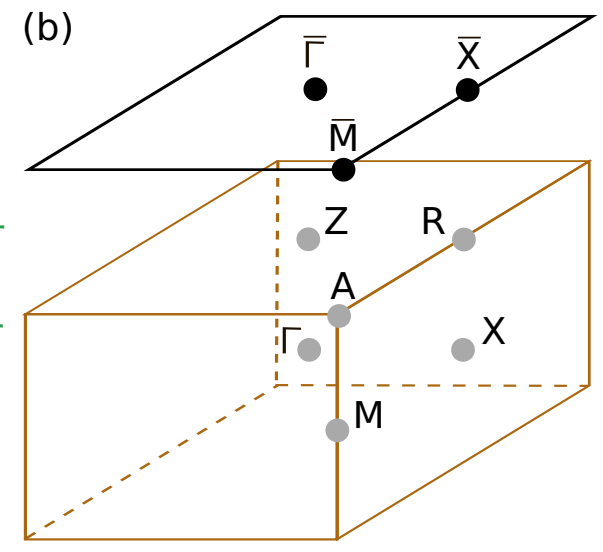

(c)

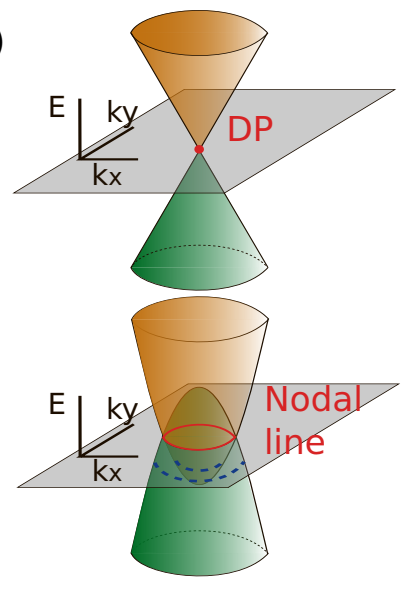

Fig. 2. (a) Crystal structure of ZrSiTe. The unit cell consists in the ordered stacking of Te (brown), Zr (green) and Si (blue) planes in quintuple layers. (b) Bulk Brillouin zone and the projected Brillouin zone on the (001) surface (SBZ), subject of our study. The high symmetry points are indicated, as well. (c) Schematics of the band dispersion in a 2D or 3D Dirac semimetal (top) and in a nodal line Dirac semimetal (bottom). In the former the Dirac Fermions form a point-like Fermi surface (DP) while in the latter the linearly dispersing states form a close-contour nodal line.

line $\overline{\Gamma X}=0.85 \AA^{-1}$. We identify several features, in particular two states, indicated by red arrows, forming diamond-like contours centered at $\bar{\Gamma}$. The origin of these two states can be understood by considering the cartoon at the bottom of Fig. 2(c). Owing to a slight doping $\mathrm{E}_{\mathrm{F}}$ intersects the band structure far from the nodal line (red), which breaks up into two distinct contours (blue dashed lines). The inner one derives from the conduction band and the outer one from the valence band. Since the real bands are more complex than the parabo- loids of Fig. 2(c) the two contours are not simply concentric circles. Nevertheless our observations are in good agreement with a recent investigation of the band structure of $\mathrm{ZrSiTe},{ }^{[52]}$ where these states were also attributed to the nodal line Dirac particles.

The two Fermi surface contours do not merge along the four equivalent $\bar{\Gamma}-\overline{\mathrm{X}} \mathrm{di}-$ rections, where hybridization opens small energy gaps. Their touching points correspond to the region of high intensity, at $\left(\mathrm{k}_{\mathrm{x}}\right.$ $\left.=0.15 \AA^{-1}, \mathrm{k}=0.55 \AA^{-1}\right)$. An additional state originating from this point, and indicated by a blue arrow, disperses towards the SBZ boundary. Notice that the intensities at symmetric points on opposite sides of $\bar{X}$ in the first and second SBZs are not identical, even if these points are formally equivalent, because the ARPES signal is modulated by k-dependent matrix element. Fig. 3(b-d) show the corresponding band dispersion along $\bar{M}-\bar{X}-\bar{M}$ (light blue line in Fig. 3(a)), measured with the He I line (a) and with $\mathrm{HHG}$ photons at $33 \mathrm{eV}$ $(\mathrm{c}, \mathrm{d})$, respectively. Interestingly, the data reveal that also this band disperses linearly over a wide energy range of almost $2 \mathrm{eV}$. Switching the light polarization from hori-

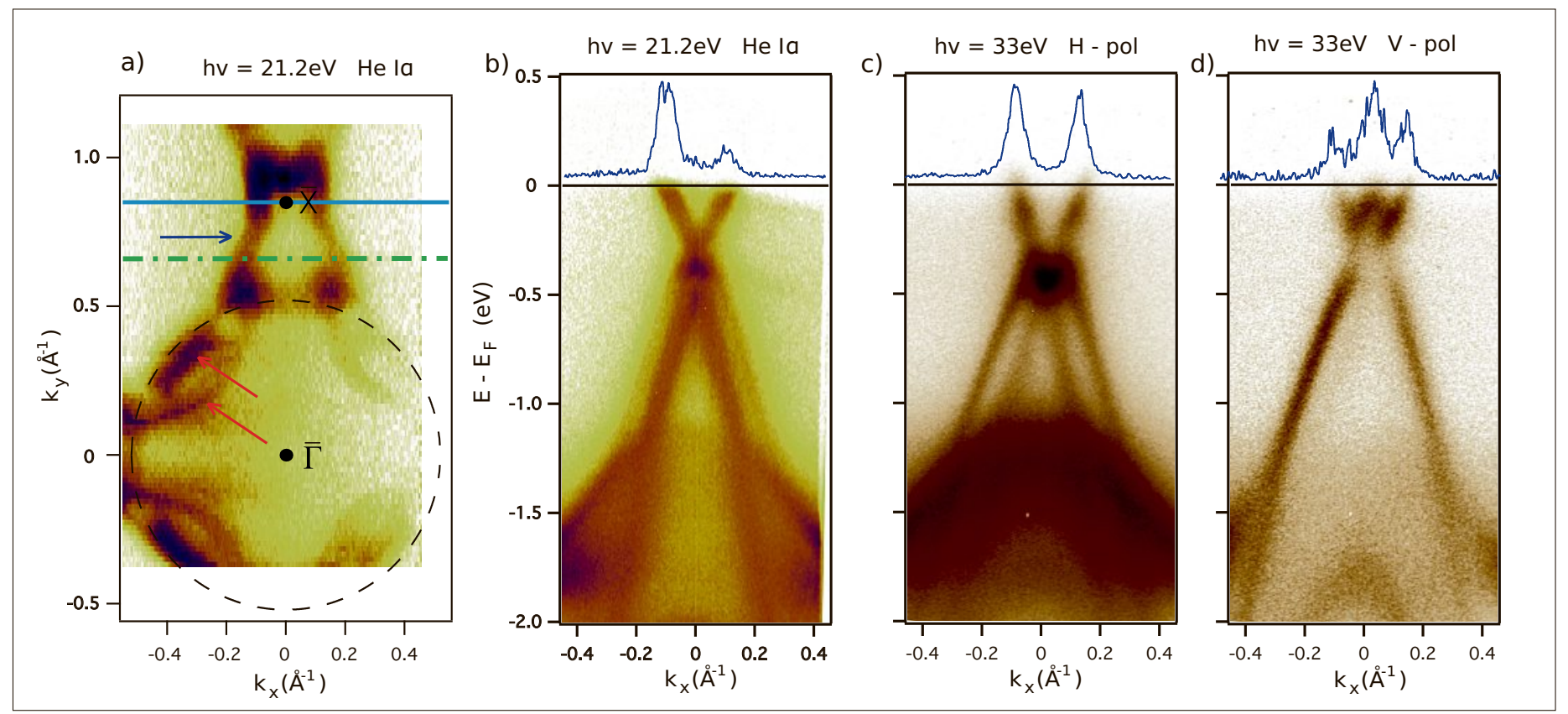

Fig. 3. (a) Fermi surface of ZrSiTe, as obtained by static ARPES mapping with the He l $\alpha$ emission line ( $h v=21.2 \mathrm{eV}$. The high-symmetry points $\bar{\Gamma}, \overline{\mathrm{X}}$ of the SBZ are shown, along with the directions where the data of Fig. 3 (blue line) and Fig. 4 (green dashed line) are taken. A dashed black circle indicates the maximum momentum window accessible at low photon energy $(5.9 \mathrm{eV}-6.2 \mathrm{eV})$ with a realistic maximum emission angle $\theta_{\max }=60^{\circ}$. (b-d) ARPES image of the band dispersion along the $\bar{M}-\bar{X}-\bar{M}$ high-symmetry directions acquired with He la emission line (a), and with HHG photons at $h v=33 \mathrm{eV}$, with horizontal (c) and vertical polarization (d). 
zontal (c) to vertical (d) provides a clearer picture of the dispersion of the Dirac cone, which is split in two, very likely under the action of spin-orbit coupling. In a centrosymmetric structure no spin splitting is possible in the bulk states. Therefore the lifting of the spin degeneracy suggests that the state at $\bar{X}$ has a surface character. ${ }^{[57]}$ This is confirmed by $a b$ initio fully relativistic calculations. ${ }^{[52]}$ We stress again that these spinsplit surface Dirac particles are only visible near $\overline{\mathrm{X}}$, at $\mathrm{k}_{\mathrm{y}}=0.85 \AA^{-1}$. Such a momentum region is precluded to tr-ARPES measurements with low-energy photons, as achieved by $4^{\text {th }}$ harmonic generation, which can only probe the momentum region inside the dashed black circle centered at $\bar{\Gamma}\left(\mathrm{k}_{\max } \approx 0.57 \AA^{-1}\right)$. Hence, a comparison between the out-of-equilibrium dynamics of the bulk nodal line Dirac particles and the spin-split surface Dirac particles in $\mathrm{ZrSiTe}$ requires VUV tr-ARPES.

During the commissioning phase of ASTRA we have performed preliminary tr-ARPES measurements of $\mathrm{ZrSiTe}$ in the region where both the bulk and surface states are present. Fig. 4(a,b) show the band dispersion for $\mathrm{k}_{\mathrm{y}}=0.65 \AA^{-1}$, along the green dashed line in Fig. 3(a). The intensity contrast in the VUV measurement at $27 \mathrm{eV}$ (b) helps to identify the contributions of the various electronic states. In particular, we attribute the intense shallow band just below $\mathrm{E}_{\mathrm{F}}$ to the surface state. The other weak spectral features arise from the bulk bands. Fig. 4(c) shows the differential ARPES image, obtained as a difference between data collected 100 fs after and $500 \mathrm{fs}$ before the arrival of the pump pulse. Blue and red indicate a reduction and, respectively, an increase of intensity. Interestingly, the optical excitation mainly affects the surface state, whereas intensity changes in the bulk states are below our present sensitivity. Fig. 4(d,e) shows the temporal evolution of the intensity averaged inside the two regions defined by red and blue rectangles in panel (b). The dynamics is fast and after 1 ps the system is back to equilibrium. This indicates the presence of a very efficient relaxation mechanism, which might go beyond the simple electron-phonon coupling. Further experiments will be necessary to elucidate it, in particular by tracking the dispersion over larger regions of the SBZ. Finally, Fig. 4(f) provides a hint of the fact that the optical excitation might not be simply described in terms of an increased electronic temperature. Energy distribution curves were integrated over a $0.2 \AA^{-1}$ wide momentum window centered at $\mathrm{k}_{\mathrm{F}}$, for negative (black) and positive (green) delay times. The depletion area below $\mathrm{E}_{\mathrm{F}}$ exceeds the positive increase above $\mathrm{E}_{\mathrm{F}}$. This observation suggests that the rapid diffusion of the optically excited carriers may yield an asymmetric distribution of hot electrons and holes. ${ }^{[25,33]}$ Further experiments are in progress to clarify this point.

In summary, we have shown that the ASTRA setup enables static and VUV time-resolved ARPES studies of solids. We illustrated its present performances with data collected on the novel Dirac semimetal compound $\mathrm{ZrSiTe}$. Detailed surveys of the equilibrium band structure can be performed with a high-brilliance monochromatized He source. They are supplemented by measurements that exploit the energy- and polarization tunability of the HHG laser-based source. We have also shown that the large-energy photons generated by Harmonium enable mapping the out-of-equilibrium dynamics in momentum regions precluded to conventional low photon energy tr-ARPES. Further investigations of the relaxation dynamics of ZrSITe will follow, with the aim to exploit circularly polarized pump pulses to optically induce an asymmetric charge population both in the bulk and surface Dirac particles.

\section{Acknowledgements}

We acknowledge financial support from the Swiss NSF via the NCCR:MUST and the contracts No. 206021-157773, and 407040154056 (PNR 70), the European Research Council Advanced Grant H2020 ERCEA 695197 DYNAMOX.

Received: April 5, 2017

[1] S. Hüfner, 'Photoelectron Spectroscopy: Principles and Applications', Springer, 2003.

[2] A. Damascelli, Z. Hussain, Z.-X. Shen, Rev. Mod. Phys. 2003, 75, 473.

[3] W. S. Fann, R. Storz, H.W. K. Tom, J. Bokor, Phys. Rev. B 1992, 46, 13592.

[4] A. H. Castro Neto, F. Guinea, N. M. R. Peres, K. S. Novoselov, A. K. Geim, Rev. Mod. Phys. 2009, 81, 109 .
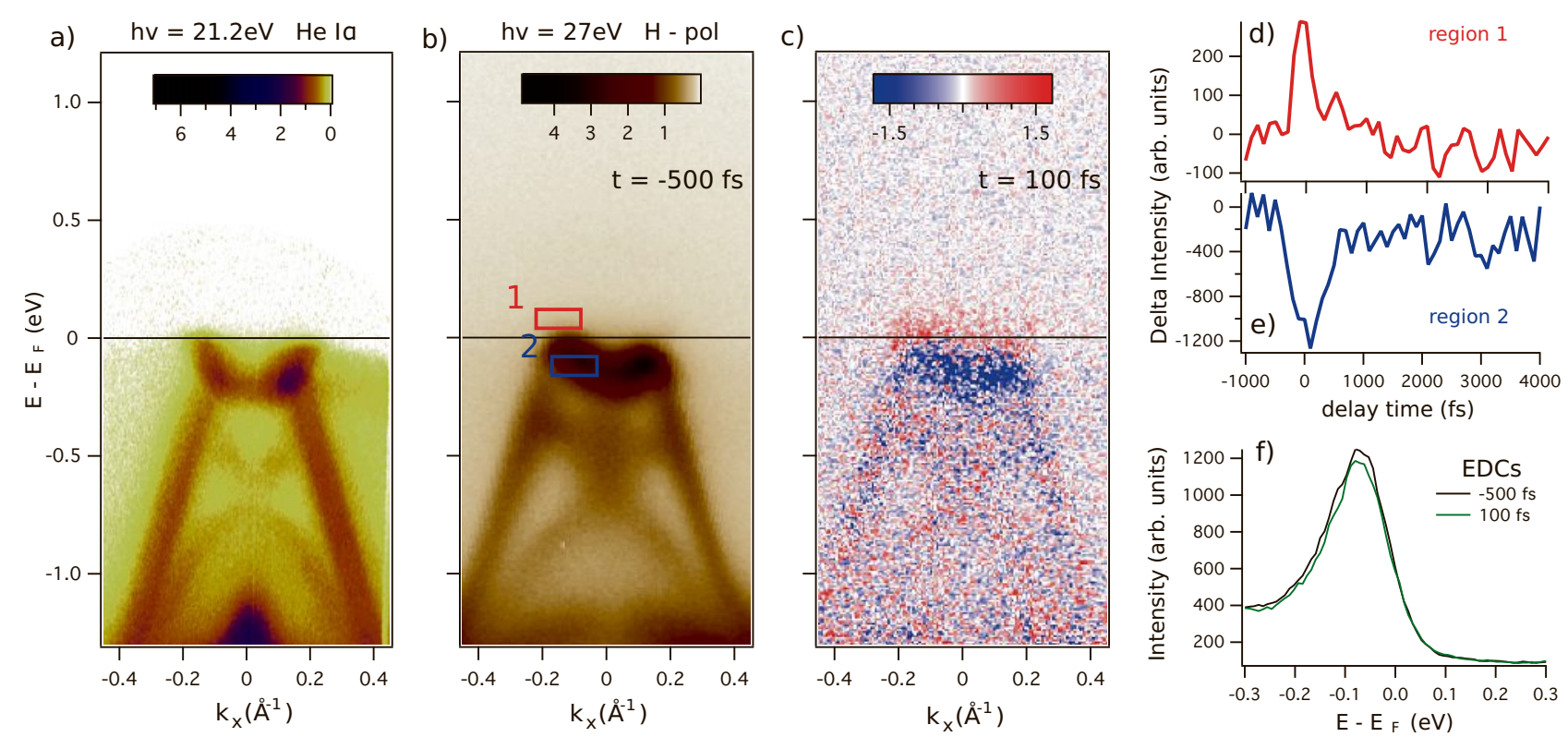

Fig. 4. (a) Band dispersion along $k_{x}$ for $k_{y}=0.65 \AA^{-1}$ (green line in Fig. 3 (a)). (b) same as in panel (a), but measured with the $H H G$ at 27 eV. (c) Differential ARPES image obtained as difference between the data $500 \mathrm{fs}$ before and $100 \mathrm{fs}$ after the arrival of the pump pulse. (d, e) Temporal dynamics of the intensity integrated in the two region within the red and blue rectangles of panel (b), respectively. (f) Comparison between the energy distribution curves measured at $-500 \mathrm{fs}$ and $100 \mathrm{fs}$. 
[5] M. Chhowalla, H. S. Shin, G. Eda, L.-J. Li, K. P. Loh, H. Zhang, Nat. Chem. 2013, 5, 263.

[6] P. R. Wallace, Phys. Rev. 1947, 71, 622.

[7] S. Y. Zhou, G.-H. Gweon, J. Graf, A. V. Fedorov, C. D. Spataru, R. D. Diehl, Y. Kopelevich, D.-H. Lee, S. G. Louie, A. Lanzara, Nat. Phys. 2006, 2,595 .

[8] A. Bostwick, T. Ohta, T. Seyller, K. Horn, E. Rotenerg, Nat. Phys. 2007, 3, 36.

[9] M. Z. Hasan, C. L. Kane, Rev. Mod. Phys. 2010 82, 3046.

[10] B.-J. Yang, N. Nagaosa, Nat. Commun. 2014, 5, 4898.

[11] T. Liang, Q. Gibson, M. N. Ali, M. Liu, R. J. Cava, N. P. Ong, Nat. Mater. 2015, 14, 280.

[12] P. Roushan, J. Seo, C. V. Parker, Y. S. Hor, D. Hsieh, D. Qian, A. Richardella, M. Z. Hasan, R. J. Cava, A. Yazdani, Nature 2009, 460, 1106.

[13] F. Bonaccorso, Z. Sun, T. Hasan, A. C. Ferrari, Nat. Photonics 2010, 4, 611.

[14] A. N. Grigorenko, M. Polini, K. S. Novoselov, Nat. Photonics 2012, 6, 749.

[15] C. X. Guo, H. B. Yang, Z. M. Sheng, Z. S. Lu, Q. L. Song, C. M. Li, Angew. Chem. Int. Ed. 2010, 49, 3014.

[16] J. W. McIver, D. Hsieh, H. Steinberg, P. JarilloHerrero, N. Gedik, Nature Nanotech. 2012, 7 , 96.

[17] H. Zeng, J. Dai, W. Yao, D. Xiao, X. Cui, Nat. Nanotech. 2012, 7, 490.

[18] Q. H. Wang, K. Kalantar-Zadeh, A. Kis, J. N Coleman, M. S. Strano, Nat. Nanotech. 2012, 7 , 699.

[19] J. A. Sobota, S. Yang, J. G. Analytis, Y. L. Chen, I. R. Fisher, P. S. Kirchmann, Z.-X. Shen, Phys. Rev. Lett. 2012, 108, 117403

[20] M. Hajlaoui, E. Papalazarou, J. Mauchain, G. Lantz, N. Moisan, D. Boschetto, Z. Jiang, I. Miotkowski, Y. P. Chen, A. Taleb-Ibrahimi, L. Perfetti, M. Marsi, Nano Lett. 2012, 12, 3532.

[21] Y. H. Wang, D. Hsieh, E. J. Sie, H. Steinberg, D. R. Gardner, Y. S. Lee, P. Jarillo-Herrero, N. Gedik, Phys. Rev. Lett. 2012, 109, 127401.

[22] A. Crepaldi, B. Ressel, F. Cilento, M. Zacchigna, C. Grazioli, H. Berger, P. Bugnon, K. Kern, M. Grioni, F. Parmigiani, Phys. Rev. B 2012, 86, 205133.

[23] M. Hajlaoui, E. Papalazarou, J. Mauchain, Z. Jiang, I. Miotkowski, Y. Chen, A. TalebIbrahimi, L. Perfetti, M. Marsi, Eur. Phys. J. Special Topics 2013, 222, 1271.

[24] A. Crepaldi, F. Cilento, B. Ressel, C. Cacho, J. C. Johannsen, M. Zacchigna, H. Berger, P. Bugnon, C. Grazioli, I. C. E. Turcu, E. Springate, K. Kern, M. Grioni, F. Parmigiani, Phys. Rev. B 2013, 88, 121404.

[25] M. Hajlaoui, E. Papalazarou, J. Mauchain, L. Perfetti, A. Taleb-Ibrahimi, F. Navarin, M. Monteverde, P. Auban-Senzier, C. Pasquier, N. Moisan, D. Boschetto, M. Neupane, M. Z. Hazan, T. Durakiewicz, Z. Jiang, Y. Xu, I Miotkowski, Y. P. CHen, S. Jia, H. W. Ji, R. J.
Cava, M. Marsi, Nat. Commun. 2014, 5, 3003.

[26] J. Sobota, S.-L. Yang, D. Leuenberger, A Kemper, J. Analytis, I. Fisher, P. Kirchmann, T. Devereaux, Z.-X. Shen, J. Electr. Spectrosc. Rel. Phenom. 2014, 195, 249.

[27] M. Neupane, S.-Y. Xu, Y. Ishida, S. Jia, B. M. Fregoso, C. Liu, I. Belopolski, G. Bian, N. Alidoust, T. Durakiewicz, V. Galitski, S. Shin, R. J. Cava, M. Z. Hasan, Phys. Rev. Lett. 2015, 115, 116801.

[28] J. C. Johannsen, G. Autès, A. Crepaldi, S. Moser, B. Casarin, F. Cilento, M. Zacchigna, H. Berger, A. Magrez, P. Bugnon, J. Avila, M. C. Asensio, F. Parmigiani, O. V. Yazyev, M. Grioni, Phys. Rev. B 2015, 91, 201101.

[29] S. Zhu, Y. Ishida, K. Kuroda, K. Sumida, M. Ye, J. Wang, H. Pan, M. Taniguchi, S. Qiao, S. Shin, A. Kimura, Sci. Report 2015, 5, 13213.

[30] K. Kuroda, J. Reimann, J. Güdde, U. Höfer, Phys. Rev. Lett. 2016, 116, 076801.

[31] A. Sterzi, A. Crepaldi, F. Cilento, G. Manzoni, E. Frantzeskakis, M. Zacchigna, E. van Heumen, Y. K. Huang, M. S. Golden, F. Parmigiani, Phys. Rev. B 2016, 94, 081111.

[32] L. Khalil, E. Papalazarou, M. Caputo, N. Nilforoushan, L. Perfetti, A. Taleb-Ibrahimi, V. Kandyba, A. Barinov, Q. D. Gibson, R. J. Cava, M. Marsi, Phys. Rev. B 2017, 95, 085118.

[33] A. Sterzi, G. Manzoni, L. Sbuelz, F. Cilento, M. Zacchigna, P. Bugnon, A. Magrez, H. Berger, A. Crepaldi, F. Parmigiani, Phys. Rev. B 2017, 95, 115431.

[34] C. Cacho, A. Crepaldi, M. Battiato, J. Braun, F. Cilento, M. Zacchigna, M. C. Richter, O. Heckmann, E. Springate, Y. Liu, S. S. Dhesi, H. Berger, P. Bugnon, K. Held, M. Grioni, H. Ebert, K. Hricovini, J. Minár, F. Parmigiani, Phys. Rev. Lett. 2015, 114, 097401.

[35] J. Sánchez-Barriga, E. Golias, A. Varykhalov, J. Braun, L. V.Yashina, R. Schumann, J. Minár, H. Ebert, O. Kornilov, O. Rader, Phys. Rev. B 2016, 93, 155426

[36] C. Jozwiak, J. A. Sobota, K. Gotlieb, A. F. Kemper, C. R. Rotund, R. J. Birgeneau, Z. Hussain, D.-H. Lee, Z.-X. Shen, A. Lanzara, Nat. Commun. 2016, 7, 13143.

[37] J. Sánchez-Barriga, M. Battiato, M. Krivenkov, E. Golias, A. Varykhalov, A. Romualdi, L. V. Yashina, J. Minár, O. Kornilov, H. Ebert, K. Held, J. Braun, Phys. Rev. B 2017, 95, 125405.

[38] J. C. Johannsen, S. Ulstrup, F. Cilento, A Crepaldi, M. Zacchigna, C. Cacho, I. C. E. Turcu, E. Springate, F. Fromm, C. Raidel, T. Seyller, F. Parmigiani, M. Grioni, P. Hofmann, Phys. Rev. Lett. 2013, 111, 027403.

[39] I. Gierz, J. C. Petersen, M. Mitrano, C. Cacho, I. C. E. Turcu, E. Springate, A. Stöhr, A. Köhler, U. Starke, A. Cavalleri, Nat. Mater. 2013, 12, 1119.

[40] S. Ulstrup, J. C. Johannsen, F. Cilento, J. A. Miwa, A. Crepaldi, M. Zacchigna, C. Cacho, R. Chapman, E. Springate, S. Mammadov, F.
Fromm, C. Raidel, T. Seyller, F. Parmigiani, M. Grioni, P. D. C. King, P. Hofmann, Phys. Rev Lett. 2014, 112, 257401.

[41] A. Stange, C. Sohrt, L. X. Yang, G. Rohde, K. Janssen, P. Hein, L.-P. Oloff, K. Hanff, K. Rossnagel, M. Bauer, Phys. Rev. B 2015, 92, 184303.

[42] J. C. Johannsen, S. Ulstrup, A. Crepaldi, F. Cilento, M. Zacchigna, J. A. Miwa, C. Cacho, R. T. Chapman, E. Springate, F. Fromm, C. Raidel, T. Seyller, P. D. C. King, F. Parmigiani, M. Grioni, P. Hofmann, Nano Lett. 2015, 15, 326.

[43] R. Bertoni, C. W. Nicholson, L. Waldecker, H. Hübener, C. Monney, U. De Giovannini, M. Puppin, M. Hoesch, E. Springate, R. T. Chapman, C. Cacho, M. Wolf, A. Rubio, R. Ernstorfer, Phys. Rev. Lett. 2016, 117, 277201.

[44] S. Ulstrup, A. G. Čabo, D. Biswas, J. M Riley, M. Dendzik, C. E. Sanders, M. Bianchi, C. Cacho, D. Matselyukh, R. T. Chapman, E. Springate, P. D. C. King, J. A. Miwa, P. Hofmann, Phys. Rev. B 2017, 95, 041405.

[45] M. Liu, M. B. Johnston, H. J. Snaith, Nature 2013, 395, 501.

[46] G. Hodes, Science 2013, 342, 317.

[47] N. J. Jeon, J. H. Noh, Y. C. Kim, W. S. Yang, S. Ryu, S. I. Seok, Nat. Mater. 2014, 13, 897.

[48] F. Cilento, G. Manzoni, A. Sterzi, S. Peli, A Ronchi, A. Crepaldi, F. Boschini, C. Cacho, R. Chapman, E. Springate, M. Capone, M. Berciu, A. F. Kemper, A. Damascelli, C. Giannetti, F. Parmigiani, arXiv:1703.03877v1, 2017.

[49] J. Ojeda, C. A. Arrell, J. Grilj, F. Frassetto, L. Mewes, H. Zhang, F. van Mourik, L. Poletto, M Chergui, Struct. Dyn. 2016, 3, 023602.

[50] L. M. Schoop, M. N. Ali, C. Strasser, A. Topp, A. Varykhalov, D. Marchenko, V. Duppel, S. S. Parkin, B. V. Lotsch, C. R. Ast, Nat. Commun. 2016, 7, 11696.

[51] J. Hu, Z. Tang, J. Liu, X. Liu, Y. Zhu, D. Graf, K. Myhro, S. Tran, C. N. Lau, J. Wei, Z. Mao, Phys. Rev. Lett. 2016, 117, 016602.

[52] A. Topp, J. M. Lippmann, A. Varykhalov, V. Duppel, B. Lotsch, C. R. Ast, L. Schoop, New J. Phys. 2016, 18, 125014.

[53] C. A. Arrell, J. Ojeda, L. Longetti, A. Crepaldi, S. Roth, G. Gatti, A. Clark, F. van Mourik, M. Drabbel, M. Grioni, M. Chergui, Chimia 2017, 71, 268.

[54] A. J. K. Haneveld, F. Jellinek, Rec. Trav. Chim. Pays-Bas 1964, 83, 776.

[55] M. N. Ali, L. M. Schoop, C. Garg, J. M. Lippmann, E. Lara, B. Lotsch, S. S. P. Parkin Sci. Adv. 2016, $2,1$.

[56] X. Wang, X. Pan, M. Gao, J. Yu, J. Jiang, J. Zhang, H. Zuo, M. Zhang, Z. Wei, W. Niu, Z. Xia, X. Wan, Y. Chen, F. Song, Y. Xu, B. Wang, G. Wang, R. Zhang, Adv. Electron. Mater. 2016, 2, 1600228.

[57] Y. A. Bychkov, E. I. Rashba, JETP Lett. 1984, 39, 78. 